\title{
If it looks and smells like the reals...
}

by

\author{
Franklin D. Tall (Toronto)
}

\begin{abstract}
Given a topological space $\langle X, \mathcal{T}\rangle \in M$, an elementary submodel of set theory, we define $X_{M}$ to be $X \cap M$ with topology generated by $\{U \cap M: U \in \mathcal{T} \cap M\}$. We prove that if $X_{M}$ is homeomorphic to $\mathbb{R}$, then $X=X_{M}$. The same holds for arbitrary locally compact uncountable separable metric spaces, but is independent of ZFC if "local compactness" is omitted.
\end{abstract}

Given a model of set theory, i.e. a collection $W$ of sets which satisfies the usual set-theoretic axioms (ZFC), a set $M \subseteq W$ is an elementary submodel of $W$ if for every natural number $n$ and for every formula $\varphi$ with $n$ free variables in the predicate calculus with $=$ and a 2 -place relation symbol $\epsilon$, and every $x_{1}, \ldots, x_{n} \in M$ (we will systematically confuse the membership relation and the symbol " $\in$ "),$\varphi\left(x_{1}, \ldots, x_{n}\right)$ holds in $M$ if and only if it does in $W$. We usually think of $W$ as being $V$, the universe of all sets, but for technical reasons officially deal with $W=H(\theta)$, the collection of all sets of hereditary cardinality less than $\theta$, a "sufficiently large" regular uncountable cardinal and rather than dealing with ZFC, we deal with sufficiently large fragments of it. (For more on these technical reasons, see [JW].) The nonlogician reader will not lose much by thinking of elementary submodels of $V$.

Elementary submodels have been used in set-theoretic topology with increasing frequency and depth over the past 20 years (see e.g. [D]). As often happens in mathematics, one's tools become objects of study; thus in $[\mathrm{JT}]$ we inaugurated a systematic investigation of the topological spaces induced by elementary submodels. This paper is a continuation of that study, although it is mainly independent of [JT].

2000 Mathematics Subject Classification: Primary 03C62, 03E35, 54A35; Secondary $54 \mathrm{~F} 65$.

Key words and phrases: elementary submodel, real line, locally compact separable metric space.

Research supported by NSERC grant A-7354. 
The Downward Löwenheim-Skolem Theorem of Logic implies that, given any set $X \in H(\theta)$ and an infinite cardinal $\kappa \leq|H(\theta)|$, there is an elementary submodel $M$ of $H(\theta)$ with $X \in M$ and $|M|=\kappa$. Given a topological space $\langle X, \mathcal{T}\rangle \in M$, we define $X_{M}$ to be the space $X \cap M$ with topology $\mathcal{T}_{M}$ generated by $\{U \cap M: U \in \mathcal{T} \cap M\}$. The Downward Löwenheim-Skolem Theorem yields $X_{M}$ 's with $X \cap M$ having any infinite cardinality $\leq|X|$; a natural question is whether an Upward Löwenheim-Skolem Theorem holds in this context, i.e. given a space $\langle X, \mathcal{T}\rangle$, is it equal or perhaps homeomorphic to $Y_{M}$ 's for $Y$ 's of arbitrary cardinality $>|X|$, and suitable $M$ 's. We shall show that this is in general false, but that it is true in some special cases. Along the way, we come across some perhaps unexpected rigidity properties of familiar spaces, e.g.

Theorem 1. If $X_{M}$ is homeomorphic to $\mathbb{R}$, so is $X$.

Subsets of $\mathbb{R}$ are sufficient to illustrate diversity with respect to such rigidity:

Theorem 2. (a) For every infinite cardinal $\kappa$, there is an $X$ of size $\kappa$ and an $M$ such that $X_{M}$ is homeomorphic to $\mathbb{Q}$.

(b) It is independent of ZFC (modulo large cardinals) whether there is an $X$ such that $X_{M}$ is homeomorphic to a subspace of $\mathbb{R}$ of size $\aleph_{1}$ but $X_{M}$ is not homeomorphic to $X$ (or even to a subspace of $\mathbb{R}$ ).

Except for some excursions into large cardinals, our proofs will use little more than the definition of elementary submodels, plus classic topology that can be found in $[\mathrm{E}]$. (We will refer to $[\mathrm{E}]$ rather than to the original authors and papers.) Thus this paper is intended to be accessible both to logicians and to topologists. Before proving a generalization of Theorem 1 we give a particularly elementary proof of the next result, which illustrates our methods.

Theorem 3. If $X_{M}$ is an uncountable compact metrizable space, then $X_{M}=X$.

Theorem 3 will be derived as a corollary to

Theorem 4. If $[0,1] \subseteq M$ and if $X_{M}$ is a hereditarily separable, hereditarily Lindelöf $T_{3}$ space, then $X_{M}=X$.

To prove Theorem 4, we need several lemmas.

Lemma 5. (a) $X_{M}$ Hausdorff implies $X$ Hausdorff.

(b) $X_{M}$ regular implies $X$ regular. 
Proof. The first is left to the reader. For the second it suffices, by elementarity, to show $M=X$ is regular, i.e. that

$$
\begin{aligned}
& (\forall x \in X \cap M)(\forall U \in \mathcal{T} \cap M)(x \in U \cap M \rightarrow(\exists V \in \mathcal{T} \cap M) \\
& (x \in V \cap M \&(\forall y \in X \cap M) \\
& [(\forall W \in \mathcal{T} \cap M)(y \in W \rightarrow W \cap V \neq \emptyset) \rightarrow y \in U])) .
\end{aligned}
$$

But since the topology on $X_{M}$ is generated by $\{U \cap M: U \in \mathcal{T} \cap M\}$, this is equivalent to saying $X_{M}$ is regular.

Lemma 6. Suppose $X, Y \in M,|X| \geq|Y|$ and $M \supseteq X$. Then $M \supseteq Y$.

Proof. There is an injection $g: Y \rightarrow X$. Hence there is an injection $g \in M$ such that $g: Y \rightarrow X$. Suppose $y \in Y$ and $x=g(y)$. Then $x \in M$ so $y$ is definable in $M$ as the unique $z$ such that $\langle z, x\rangle \in g$, so $y \in M$.

\section{Recall}

Definition. $\left\{x_{\alpha}\right\}_{\alpha<\kappa} \subseteq X$ is left-separated (resp. right-separated) if there exist open $\left\{U_{\alpha}\right\}_{\alpha<\kappa}$ such that for every $\alpha, x_{\alpha} \in U_{\alpha}$, but for all $\beta>\alpha$ (resp. $\beta<\alpha), x_{\alpha} \notin U_{\beta}$.

Lemma 7 (see e.g. $[\mathrm{R}]$ ). A space $X$ is hereditarily separable (resp. hereditarily Lindelöf) if and only if it includes no uncountable left-separated (resp. right-separated) subspace.

Lemma 8. If $\left|\omega_{1} \cap M\right|=\aleph_{1}$ and $X_{M}$ is hereditarily separable (hereditarily Lindelöf), so is $X$.

Proof. Suppose $X$ is not hereditarily separable. Then there is an injection $f: \omega_{1} \rightarrow X$ such that range $f$ is left-separated. By elementarity, there is such an $f \in M$, and if $\left|\omega_{1} \cap M\right|=\aleph_{1}$, this gives us a left-separated subspace of size $\aleph_{1}$ in $X_{M}$. Similarly for hereditarily Lindelöf.

Actually, " $\left|\omega_{1} \cap M\right|=\aleph_{1}$ " is equivalent to " $\omega_{1} \subseteq M$ ", but we do not need this here.

If $X$ is hereditarily separable (hereditarily Lindelöf), so is $X_{M}$ but we do not need this here either. [JT] is concerned with going from properties of $X$ to those of $X_{M}$; here we do the converse. Of course the difference is purely conceptual.

Proof of Theorem 4. By Lemma $6, \omega_{1} \subseteq M$ so $X$ is hereditarily Lindelöf and hereditarily separable. Since $X$ is Hausdorff by Lemma 5 (a) and hereditarily Lindelöf, $|X| \leq 2^{\aleph_{0}}$, so since $X$ is (hereditarily) separable and by 5 (b) regular, $X$ has a basis of size $\leq 2^{\aleph_{0}}$. By hereditary Lindelöfness again, $|\mathcal{T}| \leq 2^{\aleph_{0}}$, so by Lemma $6, X$ and $\mathcal{T}$ are included in $M$, so $X_{M}=X$.

Now we move on to the proof of Theorem 3 . 
Proof of Theorem 3. We use 5 classical results, two from [JT], and a new one.

Lemma 9 [E, 1.7.11]. Every uncountable compact metrizable space includes a closed dense-in-itself subspace.

Lemma 10 [E, 4.5.5(a)]. Every compact metrizable dense-in-itself space includes a copy of the Cantor set $\mathbb{K}$.

LEMma $11[\mathrm{E}, 4.5 .9(\mathrm{~b})]$. There is a continuous surjection from $\mathbb{K}$ to $[0,1]$.

LEMma 12 [E, 2.1.8]. Any continuous function from a closed subspace of a normal space into $[0,1]$ can be extended over the whole space.

Definition. A continuous function is perfect if it sends closed sets to closed sets and if each point-inverse is compact.

LEMma 13 [E, 3.7.2]. The preimage of a compact space under a perfect map is compact.

Lemma 14 [JT]. If $X$ is locally compact $T_{2}, X_{M}$ is the image of a subspace of $X$ under a perfect map.

Lemma 15 [JT]. For a first countable space $X, X_{M}$ coincides with the subspace topology on $X \cap M$.

The final lemma is due to Lucia R. Junqueira $[\mathrm{Ju}]$ and is included with her kind permission.

Lemma 16. If $X_{M}$ is compact, so is $X$.

Proof. Suppose $X$ has an open cover $\mathcal{U}$ that has no finite subcover. Then, by elementarity, there is a $\mathcal{U} \in M$ such that $M$ thinks $\mathcal{U}$ is an open cover of $X$ with no finite subcover. Then $\{U \cap M: U \in \mathcal{U} \cap M\}$ is an open cover of $X_{M}$ and hence has a finite subcover $\left\{U \cap M: U \in \mathcal{U}^{\prime}\right\}$, where $\mathcal{U}^{\prime}$ is a finite subset of $\mathcal{U}$. Then $\mathcal{U}^{\prime} \in M$ and $M$ thinks $\mathcal{U}^{\prime}$ covers $X$, so it does.

Putting these together, let $Z \subseteq X$ and $\pi: Z \rightarrow X_{M}$ be perfect and onto. Let $L \subseteq X_{M}$ be homeomorphic to $\mathbb{K}$. Let $f: L \rightarrow[0,1]$ be onto. Then, since $X$ is normal, $f \circ\left(\pi \mid \pi^{-1} L\right): \pi^{-1}(L) \rightarrow[0,1]$ extends to a $g$ mapping $X$ onto $[0,1]$. By elementarity, there is an onto $g \in M, g: X_{M} \rightarrow[0,1]_{M}$. But by Lemma $15,[0,1]_{M}=[0,1] \cap M$ with the subspace topology, so $[0,1] \cap M$ is compact. It includes $\mathbb{Q}$, so it equals $[0,1]$. Thus $[0,1] \subseteq M$.

REMARK. We should mention that although $X_{M}$ is not in general compact even if $X$ is, there are examples of $X$ 's such that $X_{M}$ is compact and yet $X_{M} \neq X$. For example, let $X$ be a one-point compactification of a discrete space and let $|M|<|X|$.

We next improve Theorem 3 to get 
THEOREM 17. If $X_{M}$ is a locally compact hereditarily Lindelöf uncountable Hausdorff space, then $X_{M}=X$.

Theorem 1 is then an immediate corollary. Indeed, any separable metrizable space is hereditarily Lindelöf.

To prove Theorem 17, we first need to show

Lemma 18. If $X_{M}$ is locally compact, so is $X$.

Proof. By elementarity, noting that finite subsets of $M$ are in $M$, it suffices to show

$$
\begin{aligned}
& (\forall x \in X \cap M)(\forall U \in \mathcal{T} \cap M)(\exists V \in \mathcal{T} \cap M)[x \in V \cap M \& \\
& (\forall y \in X \cap M)([(\forall W \in \mathcal{T} \cap M) \\
& (y \in W \cap M \rightarrow W \cap V \cap M \neq 0)] \rightarrow y \in U) \& \\
& (\forall \mathcal{S} \in M)(\mathcal{S} \subseteq \mathcal{T} \cap M \&(\forall y \in X \cap M)[(\forall W \in \mathcal{T} \cap M)(y \in W \rightarrow \\
& W \cap V \cap M \neq \emptyset) \rightarrow(\exists S \in \mathcal{S})(y \in S)] \rightarrow\left(\exists \text { finite } \mathcal{S}^{\prime} \subseteq \mathcal{S}\right) \\
& (\forall y \in X \cap M)[(\forall W \in \mathcal{T} \cap M)(y \in W \rightarrow W \cap V \cap M \neq \emptyset] \rightarrow \\
& \left.\left(\exists S \in \mathcal{S}^{\prime}\right)(y \in S)\right] .
\end{aligned}
$$

But since $X_{M}$ is locally compact, we have this.

Proof of Theorem 17. Since $X_{M}$ is locally compact and hereditarily Lindelöf, it is $\sigma$-compact and first countable. Since it is $\sigma$-compact and uncountable, it includes an uncountable compact first countable subspace. By a standard Cantor-Bendixson argument, $X_{M}$ then has an uncountable compact first countable subspace without isolated points. Such a subspace maps onto $[0,1][\mathrm{J}$, proof of 3.16]. As in the proof of Theorem 3, we then get a compact subspace $L$ of $X$ and a map $f$ from $L$ onto $[0,1]$. We then apply elementarity to

Lemma 19 [E, 3.1.C]. If $L$ is compact Hausdorff and $f: L \rightarrow Y$ is a continuous (and hence closed if $Y$ is $T_{2}$ ) surjection, then there is a closed $L^{\prime} \subseteq L$ such that $f \mid L^{\prime}$ maps $L^{\prime}$ onto $Y$ but no proper closed subset of $L^{\prime}$ is mapped by $f$ onto $Y$.

We thus get an $F \in M$ such that $F \cap M$ is closed in $X_{M}$, and there is a continuous surjection $g$ from $F \cap M$ (as a subspace of $X_{M}$ ) to $[0,1] \cap M$ such that if $H \in M$ is a closed subset of $X$, then $g(F \cap H \cap M)$ is closed in $[0,1] \cap M$, and if $g(F \cap H \cap M)=[0,1] \cap M$, then $F \cap H \cap M=F \cap M$.

$F \cap M$ is a closed subspace of a locally compact Hausdorff space and so is locally compact and hence satisfies the Baire Category Theorem. We claim $[0,1] \cap M$ does also. It suffices to show that if $V$ is dense open in $[0,1] \cap M$, then $g^{-1}(V)$ is dense open in $F \cap M$. If so, given $\left\{V_{n}\right\}_{n<\omega}$ dense open in $[0,1] \cap M$, take $x \in \bigcap_{n<\omega} g^{-1}\left(V_{n}\right)$. Then $g(x) \in \bigcap_{n<\omega} V_{n}$. To show $g^{-1}(V)$ is dense open, take $W \in \mathcal{T} \cap M$ such that $W \cap F \cap M \neq \emptyset$. If $F \cap M \subseteq$ 
$W \cap M$, then $g^{-1}(V) \cap W \cap M \neq \emptyset$, so suppose $F \cap M-W \cap M \neq \emptyset$. Then $g((F-W) \cap M) \neq[0,1] \cap M$, so there is a $y \in V \cap[0,1] \cap M-g((F-W) \cap M)$. Take $x \in F \cap M$ such that $g(x)=y$. Then $x \in g^{-1}(V) \cap W \cap M$.

$F \cap M$ is a closed subspace of a $\sigma$-compact space so it is $\sigma$-compact, say $F \cap M=\bigcup_{n<\omega} F_{n}, F_{n}$ compact. Then for some $n, g\left(F_{n}\right)$ is a compact somewhere dense subset of $[0,1] \cap M$. Therefore there are $q<r \in \mathbb{Q} \cap[0,1]$ such that $(q, r) \cap M \subseteq g\left(F_{n}\right)$. But $(q, r) \cap M$ is dense in $(q, r)$, so $g\left(F_{n}\right) \supseteq$ $[q, r]$. But then $M \supseteq[0,1]$ and we can finish off as in the proof of Theorem 3 . We need only recall that the weight (least cardinal of a base) of a locally compact Hausdorff space does not exceed its cardinality [E, 3.3.6] so "local compactness" can substitute for "hereditary separability" in Theorem 4.

REMARK. We have in effect proved that closed irreducible images of Baire spaces are Baire, as was noted in $[\mathrm{AL}]$. I thank E. Michael for supplying the reference.

After seeing this proof, S. Todorčević came up with a considerably shorter and simpler one which just uses the proof for the compact uncountable metric case, but I decided the technique of the proof given here is sufficiently interesting to justify its inclusion.

Uncountability is necessary in Theorem 3 , since if we take a countable $M$, $\left(\omega_{1}+1\right)_{M}$ is a compact metrizable space. Also observe

THEOREM 20. For any infinite regular $X$ without isolated points, there is an $M$ such that $X_{M}$ is homeomorphic to $\mathbb{Q}$.

Pr o of. Take a countable elementary submodel $M$ containing $X$. Then $X_{M}$ is regular [JT], has no isolated points, is countable, and has a countable base. But countable metric spaces without isolated points are homeomorphic to $\mathbb{Q}[\mathrm{E}, 6.2 . \mathrm{A}(\mathrm{d})]$.

Hereditary Lindelöfness - or some countability condition - is necessary in Theorem 17, else we could take the discrete space of size $\aleph_{2}$ and then take an elementary submodel of size $\aleph_{1}$. An example which is better - since $X$ has no isolated points and $M \supseteq[0,1]$ - is to take the disjoint sum of $\left(2^{\aleph_{0}}\right)^{+}$ copies of $[0,1]$ and then take a countably closed elementary submodel of size $2^{\aleph_{0}}$. Then $X_{M}$ is the sum of $2^{\aleph_{0}}$ copies of $[0,1]$, so is a locally compact uncountable metric space, but is not equal to $X$.

For general uncountable separable metric spaces, we enter the realm of large cardinals. For example,

Theorem 21. (a) If $2^{\aleph_{0}}=\aleph_{1}$ and $0^{\sharp}$ does not exist, then if $X_{M}$ is an uncountable separable metric space, $X_{M}=X$.

(b) If Chang's Conjecture holds, there is a non-metrizable $X$ such that $X_{M}$ is an uncountable separable metric space. 
$0^{\sharp}$ is a set of natural numbers, the existence of which has large cardinal strength. The non-existence of $0^{\sharp}$ is equivalent to Jensen's Covering Lemma for $L$, which is more familiar to set-theoretic topologists. $V=L$ implies $0^{\sharp}$ does not exist. See $[K]$ for details. Theorem 21(a) follows quickly from Lemma 6, Theorem 4, and

Lemma $22[\mathrm{KT}]$. If $0^{\sharp}$ does not exist and $|M| \geq \kappa$, then $\kappa \subseteq M$.

Proof of Theorem 21(a). Since $X_{M}$ and hence $M$ is uncountable, $\omega_{1} \subseteq M$. By $\mathrm{CH}$ and Lemma $6,[0,1] \subseteq M$. By Theorem 4, we are done.

Definition. Chang's Conjecture (see e.g. [K]) is the assertion that every model $M$ of size $\aleph_{2}$ with a distinguished subset $S$ of size $\aleph_{1}$ has an elementary submodel $N$ of size $\aleph_{1}$ such that $|N \cap S|=\aleph_{0}$.

Proof of Theorem 21(b). The proof divides into two cases, depending on the size of $2^{\aleph_{0}}$. First, assume $2^{\aleph_{0}} \geq \aleph_{2}$ and Chang's Conjecture. Take the Skolem Hull $M$ of a set of $\aleph_{2}$ reals in some sufficiently large $H(\theta)$. Expand $\langle M, \in\rangle$ to the model $\left\langle M, \in, \omega_{1}\right\rangle$ which distinguishes $\omega_{1}$. Take an elementary submodel $\left\langle N, \in, \omega_{1} \cap N\right\rangle$ of $\left\langle M, \in, \omega_{1}\right\rangle$ with $|N|=\aleph_{1}$ and $\left|N \cap \omega_{1}\right|=\aleph_{0}$. Now, since every member of $N$ is definable via Skolem functions from a finite set of reals, we must have $|N \cap \mathbb{R}|=\aleph_{1}$. Thus if $L$ is the Long Line, $L_{N}$ is separable, uncountable, and metrizable although $L$ is not.

Chang's Conjecture plus $2^{\aleph_{0}}=\aleph_{2}$ follows from Martin's Maximum [FMS], which is consistent if there is a supercompact cardinal. In fact, Chang's Conjecture plus the continuum arbitrary is relatively consistent from an $\aleph_{1}$-Erdös cardinal: blow up the continuum over Silver's model [S] by CCC forcing. Later, L. R. Junqueira came up with another example using the same hypothesis, which has the advantage of being compact, although it is not first countable as is the Long Line. It is simply the product of $\aleph_{1}$ copies of the two-point discrete space. When I presented my example in Toronto, S. Todorčević informed me that, using a result of Tarski [T], Baumgartner $[\mathrm{B}]$ had constructed in ZFC a linear order of density $\aleph_{1}$ and size $\aleph_{1}^{\mu}$ where $\mu$ is the least cardinal such that $\aleph_{1}^{\mu}>\aleph_{1}$. The order is obtained in the usual way from the branches of length $\mu$ of a certain tree. The corresponding linearly ordered topological space $X$ has character $\mu$; thus if $\mathrm{CH}$ holds, the space has character $\aleph_{1}$ and so is not metrizable. By taking only $\aleph_{2}$ such branches, we get $|X|=\aleph_{2}$. Applying Chang's Conjecture, we get an uncountable separable linearly ordered space $X_{M}$. This does not quite assure metrizability, but we can modify $X$ by collapsing adjacent pairs, if neither point is from the dense set $D$ of size $\aleph_{1}$. Since $D$ is dense, no point is involved in two such collapses, so the resulting space still has size $\aleph_{2}$ and its character does not change. The new $X$ will have a base of size $\aleph_{1}$ and so the resulting $X_{M}$ will have a countable base. As a bonus, it turns out 
that separable linearly ordered metrizable spaces are embeddable in $\mathbb{R}[\mathrm{E}$, 6.3.2(c)] so whether or not $\mathrm{CH}$ holds, we obtain

Corollary 23. Chang's Conjecture implies there is a non-metrizable $X$ such that $X_{M}$ is homeomorphic to a subspace of $\mathbb{R}$.

REMARK. The Long Line provides an interesting counterexample to the topological metatheorem which asserts that "homeomorphic" is the same as "equal" as far as topology is concerned. We have seen that the Long Line $L$ can have an $L_{N}$ which is homeomorphic to a subspace of $\mathbb{R}$, although $L$ is not. On the other hand, suppose we have a space $\langle X, \mathcal{T}\rangle$ such that for some $M, X_{M}$ is actually a subspace of $\mathbb{R}$. Since $\mathbb{R}$ and its topology are definable, $M \models\langle X, \mathcal{T}\rangle$ is a subspace of $\mathbb{R}$, so it is.

The conclusion of Theorem 21(a) does not follow from the non-existence of $0^{\sharp}$ :

THEOREM 24. It is consistent that $2^{\aleph_{0}}=\aleph_{2}, 0^{\sharp}$ does not exist, and there is an $M$ such that $\mathbb{R}_{M}$ is not homeomorphic to $\mathbb{R}$.

Proof. Simply add say $\aleph_{2}$ Cohen reals to a model of $V=L$. Then $0^{\sharp}$ does not exist because it cannot be added by set forcing (see e.g. [K]). Then in the extension simply take $M$ to be any elementary submodel of size $\aleph_{1}$ of some sufficiently large $H(\theta)$ including $\aleph_{1}$ reals, with $\mathbb{R} \in M$.

Under $\mathrm{CH}$, I. Farah proved all uncountable $\mathbb{R}_{M}$ 's are equal to $\mathbb{R}$. See $[\mathrm{KT}]$.

If $\left|X_{M}\right|=2^{\aleph_{0}}$, we do not need CH in the proof of Theorem 21(a) so we have e.g.

Corollary 25. If $0^{\sharp}$ does not exist and $X_{M}$ is homeomorphic to an uncountable Borel subspace of $\mathbb{R}$, then $X_{M}=X$.

I do not know if the non-existence of $0^{\sharp}$ is necessary, even for $\mathbb{R}-\mathbb{Q}$. Assuming $0^{\sharp}$ does not exist, if $\left|X_{M}\right|=\aleph_{1}$ and $X_{M}$ is separable metric, then as in the proof of Theorem 4, we see all finite powers of $X$ are hereditarily Lindelöf and hereditarily separable, so $X$ has a $G_{\delta}$-diagonal. Gary Gruenhage has shown (private communication) that nonetheless $X$ need not be metrizable.

Theorem 21(a) cannot be improved to drop separability:

EXAMPLE. It is consistent with $\mathrm{CH}$ and $0^{\sharp}$ does not exist that there is a non-metrizable first countable space of size $\aleph_{2}$ such that $X_{M}$ is metrizable for every $M$ of size $\aleph_{1}$.

Proof. $V=L$ implies there is a stationary $E \subseteq\left\{\alpha \in \omega_{2}: \operatorname{cf}(a)=\omega\right\}$ such that $E \cap \alpha$ is not stationary in $\alpha$, for any $\alpha \in \omega_{2}$. 
It follows that every subspace of $E$ of cardinality less than $\aleph_{2}$ is metrizable, but $E$ is not.

Constructing a ladder system on $E$ (see e.g. $[\mathrm{F}]$ ), one obtains a space with the additional property that it is a locally compact Moore space.

On the other hand,

Theorem 26. If for every $M$ such that $|X \cap M| \leq \aleph_{1}, X_{M}$ is separable metrizable, then $X$ is separable metrizable.

Proof. This is essentially proved in 3.2 of $[D]$. Actually, we only require one special $M$ :

Definition. $M$ is $\omega$-covering if every countable subset of $M$ is included in a member of $M$.

One can construct an $\omega$-covering elementary submodel of $H(\theta)$ with $\langle X, \mathcal{T}\rangle \in M$ as $\bigcup_{\alpha<\omega_{1}} M_{\alpha}$, where $\langle X, \mathcal{T}\rangle \in M_{0}$, a countable elementary submodel of $H(\theta), M_{\alpha+1} \supseteq M_{\alpha} \cup\left\{M_{\alpha}\right\} \cup\left\{x_{\alpha}\right\}, M_{\alpha+1}$ a countable elementary submodel of $H(\theta),\left\{x_{\alpha}: \alpha<\omega_{1}\right\} \subseteq X$, and $\alpha$ limit implies $M_{\alpha}=\bigcup_{\beta<\alpha} M_{\beta}$. Since $X_{M}$ has a countable base, there is a countable subset $\mathcal{B}$ of $\{U \cap M: U \in \mathcal{T} \cap M\}$ which is a base, since $\{U \cap M: U \in \mathcal{T} \cap M\}$ is a base. But by $\omega$-covering, we may assume $\mathcal{B} \in M$. Then $M \models X$ has a countable base, so it does.

COROLlary 27. $2^{\aleph_{0}}=\aleph_{1}$ if and only if whenever $\langle X, \mathcal{T}\rangle$ is a space such that for every $M$ such that $|X \cap M| \leq \aleph_{1}, X_{M}$ is homeomorphic to a subspace of $\mathbb{R}$, so is $X$.

Proof. Assuming $\mathrm{CH}$, since by Theorem 26, $X$ is separable metrizable and therefore $|X \cup \mathcal{T}| \leq \aleph_{1}$ we may take an $M \supseteq X \cup \mathcal{T}$ with $|M|=\aleph_{1}$. Then $X=X_{M}$ so $X$ is homeomorphic to a subspace of $\mathbb{R}$.

On the other hand, suppose $2^{\aleph_{0}}>\aleph_{1}$. Take an elementary submodel $M$ of $H(\theta)$ of size $\aleph_{1}$ containing $\mathbb{R}$. Then $(\mathbb{R} \times \mathbb{R})_{M}$ is a separable 0-dimensional metric space and hence embeddable in $\mathbb{R}$, yet $\mathbb{R} \times \mathbb{R}$ is not embeddable in $\mathbb{R}$.

An $E$ as above shows that Theorem 26 is consistently not true if one drops "separability". It will be difficult to construct just in ZFC a nonmetrizable $X$ such that all $X_{M}$ with $|X \cap M| \leq \aleph_{1}$ are metrizable. The reason is that L. R. Junqueira has shown that if there is an $\omega$-covering $M$ such that $X_{M}$ is metrizable, then $X$ is first countable and all subsets of size $\leq \aleph_{1}$ are metrizable [Ju]. No example in ZFC is known of such a non-metrizable $X$.

Assuming the existence of a supercompact cardinal, there is a topological Upward Löwenheim-Skolem Theorem for large spaces:

TheOREM 28. Suppose $|X| \geq \kappa$, a supercompact cardinal, say $\langle X, \mathcal{T}\rangle$ $\in H(\theta)$. Then for every $\lambda \geq \theta+|X \cup \mathcal{T}|$ there is a $\langle Y, \mathcal{S}\rangle,|Y| \geq \lambda$, a $\Psi$ and 
an elementary submodel $M$ of $H(\Psi)$ such that $\langle Y, \mathcal{S}\rangle \in M$ and $\langle X, \mathcal{T}\rangle$ is homeomorphic to $Y_{M}$.

Proof. Take a supercompact embedding $j$ with $j(\kappa) \geq \lambda$. Take $M=$ $j^{\prime \prime} H(\theta)$. Then $M$ is an elementary submodel of $H(j(\theta))$. Let $Y=j(X)$ and $\mathcal{S}=j(\mathcal{T})$. Then $\langle X, \mathcal{T}\rangle$ is homeomorphic to $Y_{M}$, which is just $j^{\prime \prime} X$ with topology $\left\{j^{\prime \prime} U: U \in \mathcal{T}\right\}$.

One can attempt to carry out this construction in case we have a sufficiently closed elementary embedding $j$ existing in some generic extension of $V$. In this case, however, $Y_{M} \notin V$ and is no longer homeomorphic to $\langle X, \mathcal{T}\rangle$ - which is no longer a topological space - but rather to the topology on $X$ generated by $\mathcal{T}$ in that generic extension.

Let me end by restating the most interesting remaining open problem:

Problem. Is it a theorem of ZFC that if $X_{M}$ is homeomorphic to $\mathbb{R}-\mathbb{Q}$, then $X=X_{M}$ ?

This paper was written while the author was an Honorary Fellow at the University of Wisconsin (Madison) in the summer of 1997. He thanks the members of the Department for their hospitality. He also thanks Ernest Schimmerling, Menachem Magidor, Mirna Džamonja, Kenneth Kunen, Stevo Todorčević, and Ilijas Farah for conversations concerning the subject of the paper, and the referee for catching some errors in an earlier version.

\section{References}

[AL] J. M. Aarts and D. J. Lutzer, Completeness properties designed for recognizing Baire spaces, Dissertationes Math. 116 (1974).

[B] J. E. Baumgartner, Almost disjoint sets, the dense set problem and partition calculus, Ann. Math. Logic 10 (1976), 401-439.

[D] A. Dow, An introduction to applications of elementary submodels in topology, Topology Proc. 13 (1988), 17-72.

[E] R. Engelking, General Topology, Heldermann, Berlin, 1989.

[F] W. G. Fleissner, On $\lambda$-collectionwise Hausdorff spaces, Topology Proc. 2 (1977), 445-456.

[FMS] M. Foreman, M. Magidor and S. Shelah, Martin's Maximum, saturated ideals, and non-regular ultrafilters, I, Ann. of Math. 127 (1988), 1-47.

[J] I. Juhász, Cardinal Functions in Topology - Ten Years Later, Math. Centre, Amsterdam, 1980.

[Ju] L. R. Junqueira, Upwards preservation by elementary submodels, preprint.

[JT] L. R. Junqueira and F. D. Tall, The topology of elementary submodels, Topology Appl. 82 (1998), 239-266.

[JW] W. Just and M. Weese, Discovering Modern Set Theory, Volume II, Amer. Math. Soc., Providence, 1997.

[K] A. Kanamori, The Higher Infinite, Springer, Berlin, 1994. 
[KT] K. Kunen and F. D. Tall, The real line in elementary submodels of set theory, J. Symbolic Logic, to appear.

[R] J. Roitman, Basic S and L, in: Handbook of Set-Theoretic Topology, K. Kunen and J. E. Vaughan (eds.), North-Holland, Amsterdam, 1984, 295-326.

[S] J. H. Silver, The independence of Kurepa's conjecture and two-cardinal conjectures in model theory, in: Axiomatic Set Theory, D. S. Scott (ed.), Proc. Sympos. Pure Math. 13, Amer. Math. Soc., Providence, 1971, 383-390.

[T] A. Tarski, Sur la décomposition des ensembles en sous-ensembles presque disjoints, Fund. Math. 12 (1928), 188-205.

Department of Mathematics

University of Toronto

Toronto, Ontario, Canada M5S 3G3

E-mail: tall@math.toronto.edu

Received 1 November 1998;

in revised form 10 August 1999 